(2) Open Access Full Text Article

ORIGINAL RESEARCH

\title{
Genipin Induces Autophagy and Suppresses Cell Growth of Oral Squamous Cell Carcinoma via PI3K/AKT/MTOR Pathway
}

This article was published in the following Dove Press journal:

Drug Design, Development and Therapy

\author{
MingBo Wei' \\ YanLi Wu ${ }^{2}$ \\ Hui Liu' \\ Chun $\mathrm{Xie}^{3}$ \\ 'Department of Stomatology, Zhongnan \\ Hospital of Wuhan University, Wuhan, \\ Hubei, 43007I, People's Republic of \\ China; ${ }^{2}$ Department of Pharmacy, \\ Zhongnan Hospital of Wuhan University, \\ Wuhan, Hubei, 43007I, People's Republic \\ of China; ${ }^{3}$ Stomatology Center, Affiliated \\ Union Hospital of Tongji Medical College, \\ Huazhong University of Science and \\ Technology, Wuhan, Hubei, 430022, \\ People's Republic of China
}

Background: Oral squamous cell carcinoma (OSCC) is a common malignant tumor of the head and neck, and it accounts for more than $90 \%$ of oral cancer. Due to high mortality, limitations of traditional treatment and many complications, new treatment methods are urgently needed. This study aimed to look into the effect of new potential anti-tumor drug, genipin, on OSCC treatment. Methods: In vitro, $\mathrm{CCK}-8$, colony formation, and flow cytometry were used to detect the effect of genipin on SCC-9 and SCC-15 cell lines. Immunofluorescence, real-time PCR, and Western blotting were used to investigate its mechanism. Xenograft tumor model was used to explore the role of genipin in vivo.

Results: We found that genipin suppressed cell growth and induced apoptosis in vitro. In addition, the expression of p62 was down-regulated while Beclin1 and LC3II were up-regulated in SCC-25 and SCC-9 cells. 3-methyladenine (3-MA) significantly decreased LC3 $(\mathrm{LC} 3 \mathrm{II})^{+}$puncta, but genipin rescuect $3 \mathrm{~d}$ this reduction. Furthermore, genipin also reduced the expression of p-PI3K, p-AKT, and p-mTOR. In vivo experiment showed that genipin significantly curbed the tumor size and weight. The positive expression of Ki67 protein and number of apoptotic cells were increased.

Conclusion: Conclusively, this study implicated that genipin suppresses cell proliferation and stimulated apoptosis, and is the first exploration showing that genipin induces OSCC cell autophagy via PI3K/AKT/mTOR pathway inhibition.

Keywords: genipin, oral squamous cell carcinoma, autophagy, PI3K/AKT/mTOR pathway

\section{Introduction}

Oral cancer is an increasing threat worldwide, with around 640,000 new cases being detected annually globally. ${ }^{1,2}$ In addition, there are 145,000 deaths per year in oral cancer, ${ }^{3}$ and five-year survival rates of patients with advanced oral cancer are $30-40 \%$. ${ }^{4}$ Owing to the lack of early symptoms and appropriate screening for precancerous lesions, most oral cancer cases are not detected until later stages. The incidence of oral cancer is mainly associated with the consumption of areca nut, tobacco, and alcohol. ${ }^{5}$ Notably, oral squamous cell carcinomas (OSCC) account for more than $90 \%$ of oral cancer and is more likely to occur in men, the risk increases with age. ${ }^{6}$ Tongue is the most common site of OSCC, which has little effect on other parts of the mouth. So far, the mechanism of oral squamous cell carcinoma has not been fully elucidated, which seriously restricts the prognosis and treatment strategy.
Stomatology Center, Affiliated Union Hospital of Tongji Medical College, Huazhong University of Science and Technology, NO. 1277 Jiefang Avenue, Wuhan 430022, Hubei, People's Republic of China

Tel +8627-84309882

Email alaw9vf@sina.com 
Genipin, an aglycone of geniposide, is a major component of the fruit of Gardenia jasminoides, ${ }^{7}$ and it is an iridoid glycoside hydrolyzed from intestinal microbiota. ${ }^{8}$ Genipin has been used in the pharmacological treatment of cardiovascular disorders, such as xenograft scaffolds of heart valves. ${ }^{9}$ Moreover, Genipin is also considered to be a therapeutic drug for liver fibrosis. ${ }^{10}$ Previous studies show that Genipin has a wide range of anticancer activities in different types of human cancer. Parallelly, several reports have suggested that Genipin can inhibit cell invasion and migration, ${ }^{11}$ and induce apoptosis ${ }^{12}$ of colon cancer cells. ${ }^{13}$ However, the protective effect of Genipin on oral carcinoma is still ambiguous.

PI3K/AKT/mTOR signaling pathway plays hub roles in cell proliferation, angiogenesis, transcription, and translation. ${ }^{14,15}$ Substantially, PI3K is a phosphoinositide kinase that catalyzes PIP2 phosphorylation when activated by $\mathrm{G}$ protein conjugated and tyrosine kinase receptors. ${ }^{16}$ Consequently, phosphorylated PIP2 then further phosphorylate AKT and stimulate downstream mTOR, eventually activating protein translation and promote cell growth or proliferation. ${ }^{17}$ Besides, abnormal PI3K/AKT/mTOR pathway causes many human cancers, including human oral cancer. ${ }^{18}$ Therefore, PI3K/AKT/mTOR pathway maybe is a crucial target against cancer.

In this study, we explored the effect of Genipin on OSCC and tried to provide theoretical basis for drug treatment of cancer.

\section{Materials and Methods}

\section{Cell Culture and Genipin Treatment}

Normal human oral epithelial cells NHOK and human tongue squamous cancer cells SCC-25 and SCC-9 were obtained from Procell Life Science\&Technology Co., Ltd (Wuhan, China), and were cultured in DMEM (Thermo Fisher Scientific, Waltham, USA) medium and supplemented with $10 \%$ fetal bovine serum (FBS, Gibco, Shanghai, China), penicillin $(100 \mathrm{U} / \mathrm{mL})$, and streptomycin $(100 \mu \mathrm{g} /$ $\mathrm{mL})$ at $37^{\circ} \mathrm{C}$ with $5 \% \mathrm{CO}_{2}$. Then, cells were treated with Genipin (King Tiger, Chengdu, China), which was dissolved in $0.1 \%$ dimethyl sulfoxide (DMSO, SigmaAldrich, Shanghai, China). Cells treated with DMSO alone were set as control.

\section{Cell Viability Assay}

NHOK, SCC-25, and SCC-9 cells suspension were seeded in 96-well plate $(100 \mu \mathrm{L} /$ well $)$, respectively. Then, the plates were placed in the incubator $\left(37^{\circ} \mathrm{C}, 5 \% \mathrm{CO}_{2}\right)$ for pre-culture. Next, $10 \mu \mathrm{L}$ of CCK-8 solution (Beyotime, Shanghai, China) was added into each well according to the manufacturer's instructions, and incubated for $48 \mathrm{hrs}$ with different concentrations of Genipin ( 0 to $400 \mu \mathrm{M})$. The absorbance at $450 \mathrm{~nm}$ was detected by a microplate reader (Bio-Rad, Hercules, CA, USA) and the changes of cell viability were estimated.

\section{Clone Formation Assay}

Firstly, about $3 \times 10^{2}$ SCC-25 and SCC-9 cells were treated by Genipin with three different concentrations $(10,30,60 \mu \mathrm{M})$ for $72 \mathrm{hrs}$, respectively. Next, cells were seeded into $35 \mathrm{~mm}$ dishes and cultured for 14 days. After that, two types of cells were rinsed with PBS and fixed in 4\% paraformaldehyde (Sigma, USA) for 15 mins at room temperature, respectively. Then, they were incubated with Wright-Giemsa dye for 5 mins with Wright-Giemsa stain (NJJCBIO, Nanjing, China) for 5 mins. Finally, the images of clone formation were captured and number of cell clones were calculated.

\section{Flow Cytometry}

Briefly, SCC-25 and SCC-9 cells were fixed for $24 \mathrm{hrs,}$ respectively. Next, the fixed cells were stained with an Annexin V-FITC Apoptosis Detection Kit (Beyotime, Shanghai, China) according to the manufacturer's instructions. After gently mixing, cells were incubated at room temperature for 20 mins in dark. Finally, red fluorescence was detected by flow cytometry (Thermo Fisher Scientific, Waltham, USA) at $488 \mathrm{~nm}$ wavelength.

\section{Real-Time PCR}

Total RNA of SCC-25 and SCC-9 cells was isolated using TRIzol reagent (Invitrogen, Waltham, USA) and cDNA was synthesized by a PrimeScript RT Reagent kit (TaKaRa, Kusatsu, Japan), according to manufacturer's instructions. Next, Real-time PCR was performed using a SYBR Green Master mix (TaKaRa, Kusatsu, Japan), including $1 \mu \mathrm{L}$ primers $(10 \mu \mathrm{mol} / \mathrm{L})$ and $1 \mu \mathrm{L} \mathrm{cDNA}(100 \mathrm{ng} / \mu \mathrm{L}) . \beta$-actin was used as endogenous control and relative mRNA levels normalized by $\beta$-actin were calculated employing $2^{-\Delta \Delta C t}$ mean. The primers sequences are listed below: Ki67-F: 5'-GCAGGACTTCAC TTGCTTCC-3, Ki67-R: 5'-TCATTTGCGTTTGTTTCACG -3'; Survivin-F: 5'-GACCACCGGATCTACACCTT-3', Survi vin-R: 5'-TCTTCCACCTGCTTCTTGACT-3'; $\beta$-actin-F: 5'CTGTCTGGCGGCACCACCAT-3', $\beta$-actin-R: 5'-GCAACT AAGTCATAGTCCGC - $3^{\prime}$. 


\section{Western Blotting}

Total protein was extracted from SCC-25 and SCC-9 cells using Radioimmunoprecipitation (RIPA) Assay Buffer (Thermo Fisher Scientific, Waltham, USA). And the protein lysates were separated on SDS-PAGE gel and blotted onto polyvinylidene difluoride (PVDF, Millipore, Boston, USA) membranes. Next, the primary antibody was incubated after blocking in 5\% non-fat milk. All antibodies were obtained from the Abcam (Cambridge, UK) and shown below: Ki67 (ab16667, 1:1000), survivin (ab76424, 1:1000), cleaved PARP1 (ab32561, 1:1000), cleaved caspase-3 (ab2302, 1:1000), P62 (ab109012, 1:1000), Beclin1 (ab207612, 1: 1000), LC3I (ab192890, 1:1000), LC3II (ab52628, 1:1000), PI3K (ab70912, 1:1000), p-PI3K (ab18265, 1:1000), AKT (ab179463, 1:1000), p-AKT (ab38449, 1:1000), mTOR (ab2 732, 1:1000), p-mTOR (ab109268, 1:1000), and $\beta$-actin (ab179467, 1:1000). Finally, the strips were exposed with ECL reagent (Sangon Biotech, Shanghai, China) following by incubating with a horseradish peroxidase-conjugated secondary antibody.

\section{Immunofluorescence Staining}

SCC-25 and SCC-9 cells were grown on glass coverslip and cultured with or without Genipin $(10,30$, and $60 \mu \mathrm{M})$, respectively. After washing twice in PBS, the cells were fixed with $4 \%$ paraformaldehyde for 20 mins. Then, cells were permeabilized for 10 mins in PBS containing $0.1 \%$ Triton X-100 (Sigma-Aldrich, Shanghai, China). Next, they were blocked in $4 \%$ goat serum blocking solution for 30 mins at room temperature. Afterwards, the cells were incubated with LC3 antibody at $4^{\circ} \mathrm{C}$ overnight. On the following day, the fluorescein-labeled secondary antibody were added and incubated in darkness at $37^{\circ} \mathrm{C}$ for $60 \mathrm{mins}$. Finally, the images were observed and photographed under a fluorescence microscope (Carl Zeiss, Jena, Germany).

\section{Animal Experiments}

Male BALB/c nude mice (4-6 weeks old, $20 \pm 2$ g) were obtained from the Animal Center of the Wuhan University Zhongnan Hospital (Wuhan, China). Mice were housed 6 per cage at $25^{\circ} \mathrm{C}$ and $80 \%$ humidity in a $12 \mathrm{hrs}$ light/ dark cycle for free access to food and water. OSCC xenograft model was established in mice by subcutaneous injection of SCC-25. When the tumor volume reached $100 \mathrm{~mm}^{3}$, $30 \mathrm{mg} / \mathrm{kg}^{19}$ Genipin was injected intraperitoneal for a week. Meanwhile, the control $(\mathrm{n}=6)$ was injected with $0.9 \% \mathrm{NaCl}$. At 30 days, the tumors were weighed and volume were measured. All animal experiments and operations were implemented in accordance with the guidelines of the National Institute of Laboratory Animal Care and Use (8th edition, National Academy of Sciences Press, 2011), and approved by the ethics committee of Wuhan University Zhongnan Hospital.

\section{Histopathological Analysis}

Histopathological assays were performed using fresh mice tissue. In brief, after the mice were sacrificed, tumor and normal tissues were fixed in formalin and paraffin embedded. The paraffin sections were sliced to $4 \mu \mathrm{m}$ thick. According to the manufacturer's instructions, the positive expression of Ki67 was detected using a DAB Substrate kit (ZSGB-BIO Co., Ltd., beijing, China) and the number of apoptosis was detected by a One-Step TUNEL Apoptosis Assay Kit (Beyotime, Shanghai, China). Antibodies were obtained from the Abcam (Cambridge, UK).

\section{Statistical Analysis}

Data are presented as the mean \pm standard deviation (SD), and IBM SPSS Statistics 25.0 was used for statistical analysis (IBM, Armonk, USA). One-way ANOVA analysis of variance was conducted to analyze the significance of differences. Multiple comparisons using one-way analysis of variance and Newman-Keuls test. $\mathrm{P}<0.05$ was considered to indicate a statistically significant difference.

\section{Results \\ Genipin Inhibited Viability of Oral Carcinoma Cells}

Base on the toxic effect of Genipin in colon cancer, ${ }^{19}$ CCK8 assay were conducted to measure the cell activity using a concentration gradient of Genipin $(0$ to $400 \mu \mathrm{M})$. As shown in Figure 1B, we found that Genipin inhibited SCC25 and SCC-9 cells in a concentration-dependent manner, but when the concentration was below $100 \mu \mathrm{M}$, there was no significant effect on normal oral epithelial cells (NHOK). In addition, when the concentration were $\geq 60 \mu \mathrm{M}$, Genipin obviously suppressed the viability of SCC-25 and SCC-9 cells. Given this, we selected three concentrations of 10 , 30 , and $60 \mu \mathrm{M}$ for subsequent in vitro experiment.

\section{Genipin Suppressd OSCC Cell Growth}

According to the clone formation experiment, Genipin concentration-dependent inhibited the growth of OSCC cells (Figure 2A). When Genipin exceeded $30 \mu \mathrm{M}$, the cell colony 
A

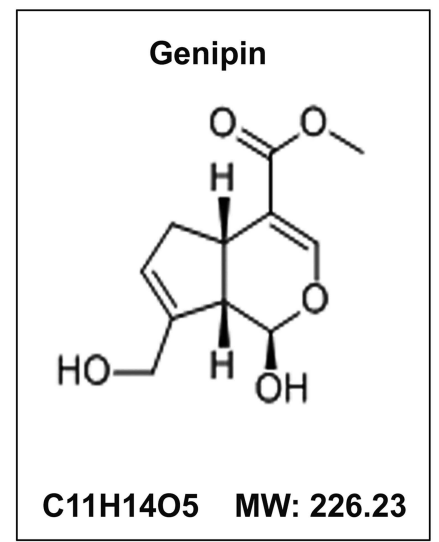

B

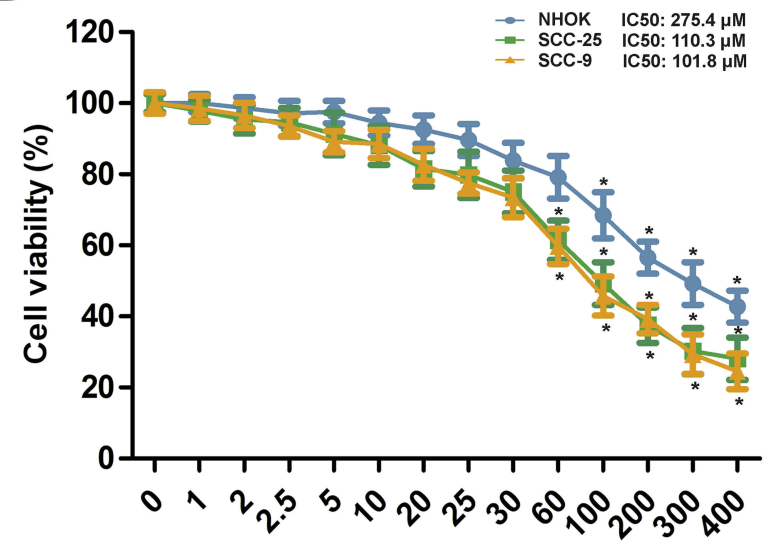

Figure I Effect of Genipin on the viability of human oral cells. (A) The chemical structure of Genipin and its molecular weight (MW). (B) CCK8 assay were conducted to measure cell viability of normal human oral epithelial cells NHOK, human tongue squamous cancer cells SCC-25 and SCC- 9 with different concentration of Genipin ( 0 to $400 \mu \mathrm{M}) .{ }^{*} \mathrm{p}<0.05$ compared with 0 . Data are presented as mean $\pm \mathrm{SD}$, and all the experimental in triplicate.

formation rate was significantly reduced compared with the control group. In addition, we also detected the expression of ki67 (proliferation maker) and Survivin (apoptosis maker) by $\mathrm{qPCR}$ and Western blotting, respectively. As shown in Figure 2B, the mRNA and protein levels of Ki67 and Survivin were decreased by the treatment of Genipin in SCC-25. Parallelly, the result in SCC-9 cells was similar to SCC-25 (Figure 2B). Hence, the above data suggested that Genipin suppressed OSCC cell proliferation.

\section{Genipin Induced OSCC Cell Apoptosis}

In order to further explored the effect of Genipin on cell proliferation, we detected cell apoptosis using flow cytometry. We found that the apoptosis rate of SCC-25 and SCC-9 cells significantly increased after Genipin treatment (10, 30, and $60 \mu \mathrm{M}$ ), compared with control (Figure 3A and $\mathrm{B}$ ). Moreover, the marked protein (caspase-3 and PARP) of apoptosis were detected by Western blotting, and the data showed that the protein levels of cleavedcaspase-3 and cleaved-PARP were obviously elevated after Genipin treatment (Figure 3C and D). These results confirmed that Genipin promoted cell apoptosis in OSCC.

\section{Genipin Promoted OSCC Cell Autophagy}

In order to study the effect of Genipin on autophagy, we detected the related proteins expression of autophagy in SCC-25 and SCC-9 cells through Western blotting. The results showed that Genipin significantly down-regulated the expression of p62 and upregulate the expression of Beclin 1 and LC3II/LC3I in a concentration-dependent manner (Figure 4A and $\mathrm{B})$. In addition, immunofluorescence assay showed that intracellular LC3 (LC3II) ${ }^{+}$puncta increased significantly after Genipin treatment (Figure 4C). Besides, autophagy process was further confirmed by autophagy inhibitor 3-methyladenine (3-MA, $5 \mathrm{mM}$ ), we found that 3-MA significantly inhibited $\mathrm{LC}^{+}$puncta, and Genipin weakened this trend after combined treatment with Genipin and 3-MA (Figure 4D).

\section{$\mathrm{PI}$ KK/AKT/mTOR Pathway Was Involved in the Anticancer Effect of Genipin on OSCC Cell}

Since the abnormal regulation of PI3K/AKT/mTOR signaling pathway is very common in human cancer, we have explored whether the Genipin regulated this pathway in human OSCC. As shown in Figure 5A and C, the phosphorylation levels of PI3K, AKT, and mTOR were downregulation after treated with Genipin. Then, we added PI3K activator 740Y-P $(20 \mu \mathrm{M})$. As shown in Figure 5B and D, $740 \mathrm{Y}-\mathrm{P}$ increased the expression of p-PI3K, p-AKT, and p-mTOR, as well as the protein levels of cleaved-caspase- 3 and LC3II. But, after co-treatment with Genipin and 740Y-P, Genipin weakened the activation of the pathway by 740Y-P.

\section{Genipin Alleviated OSCC Tumor in vivo}

Combined with the results of in vitro experiments, we established a SCC-25 xenograft tumor model. The data showed that compared with the control group, the tumor weight and the tumor volume in the treatment group were significantly reduced (Figure 6A-C). Immunohistochemistry assay showed that the expression of Ki67 was down-regulation (Figure 6D), and TUNEL assay manifested that the number of apoptosis was increased in tumor tissue (Figure 6D). In 


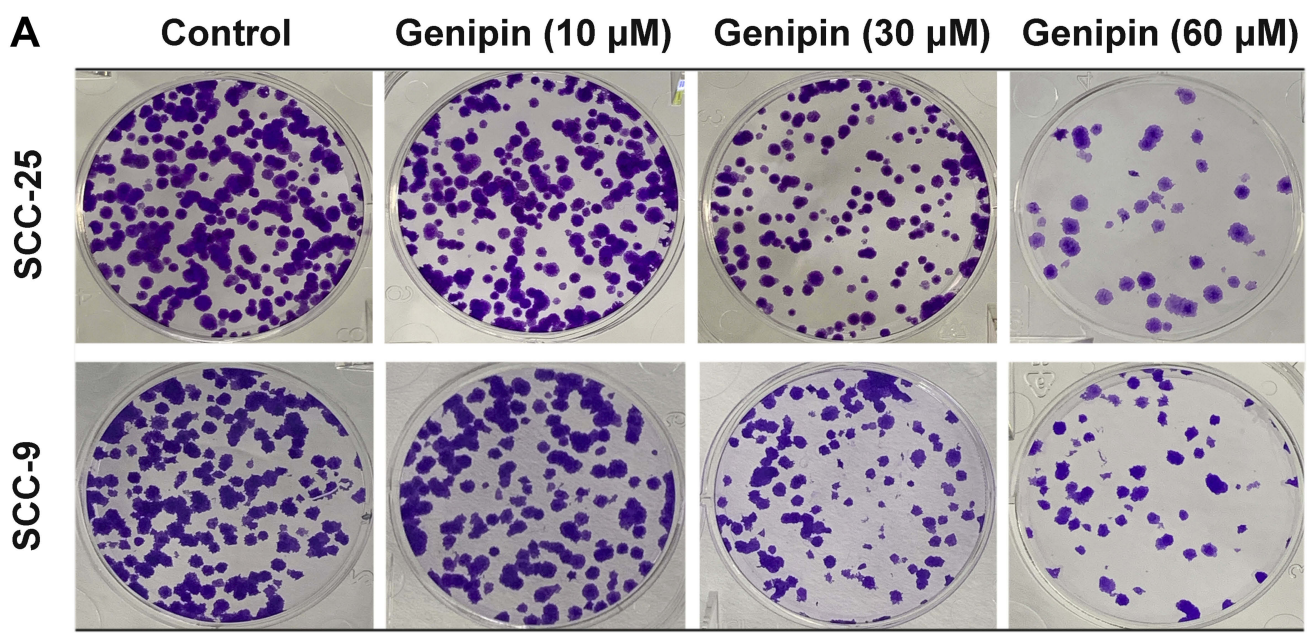

B

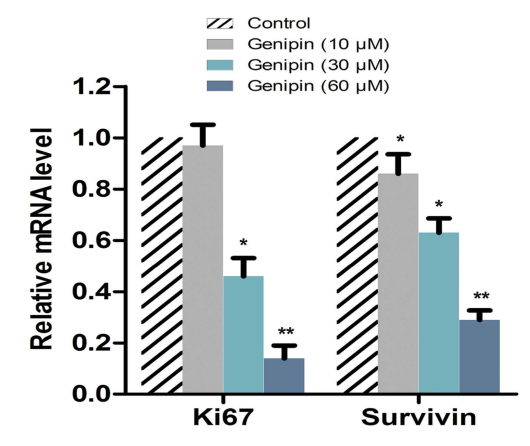

C

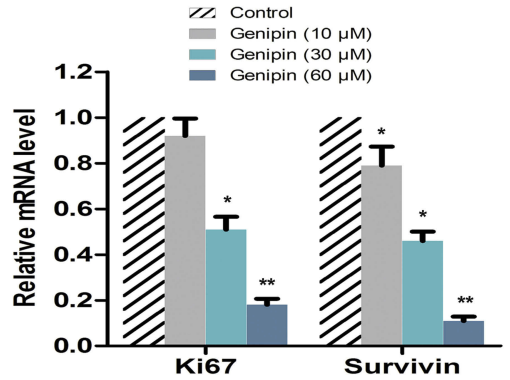

SCC-25

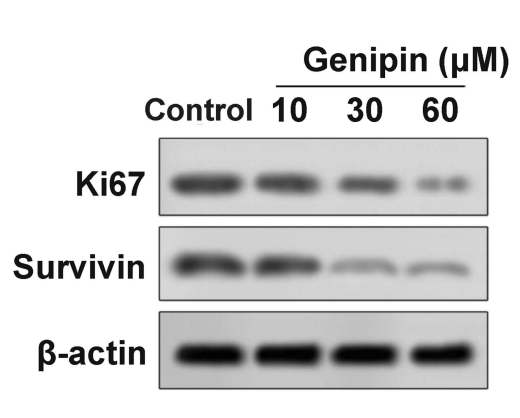

SCC-9

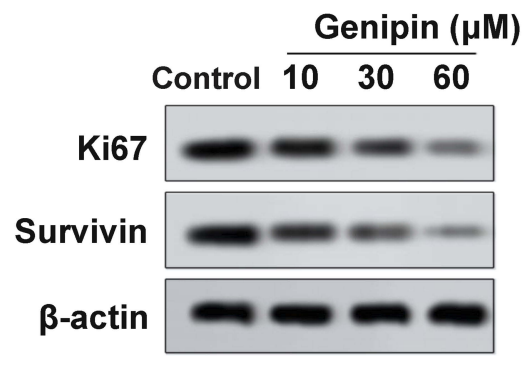

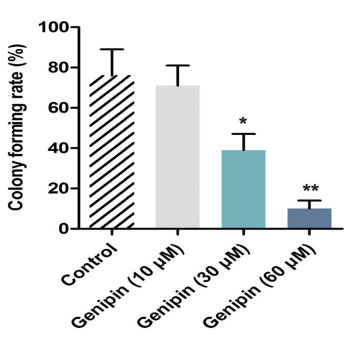

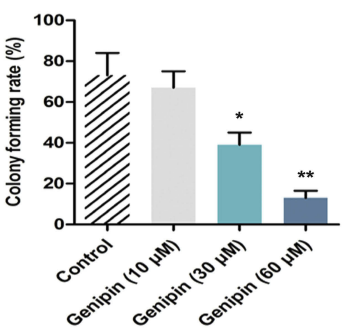

Figure 2 Effect of Genipin on cell growth in OSCC. SCC-25 and SCC-9 cells were treated with three concentrations of Genipin (I0, 30 , and $60 \mu$ M). (A) SCC-25 and SCC9 cells proliferation ability were measured by clone formation assay. (B) The mRNA and protein levels of Ki67 and Survivin in SCC-25 were detected by qPCR and Western blotting, respectively. (C) The mRNA and protein levels of Ki67 and Survivin in SCC-9 were detected by qPCR and Western blotting, respectively. ${ }^{*}<0.05$, ** $<<0.01$ compared with the control group. Data are presented as mean $\pm \mathrm{SD}$, and all the experimental in triplicate.

addition, we also found that the protein levels of cleavedcaspase-3 and LC3II were increased in the Genipin treatment group, compared to the control (Figure 6E).

\section{Discussion}

At present, OSCC remains a major threat to increased morbidity and poor 5-year survival. ${ }^{2}$ Although OSCC has advantages of timely treatment due to relatively easier access for early diagnosis, it is also an aggressive disease with local recurrence and cervical lymph node metastasis. ${ }^{2}$ The basic method of therapy includes surgical resection leaving a margin of healthy tissue, and neck dissection during node metastases. ${ }^{20,21}$ However, conventional therapy often leads to impaired oral functions. $^{22}$ Moreover, radiotherapy often leads to several of complications such as xerostomia, radiation caries, taste disorders, and mucositis. ${ }^{23-25}$ This study provided insight into the 

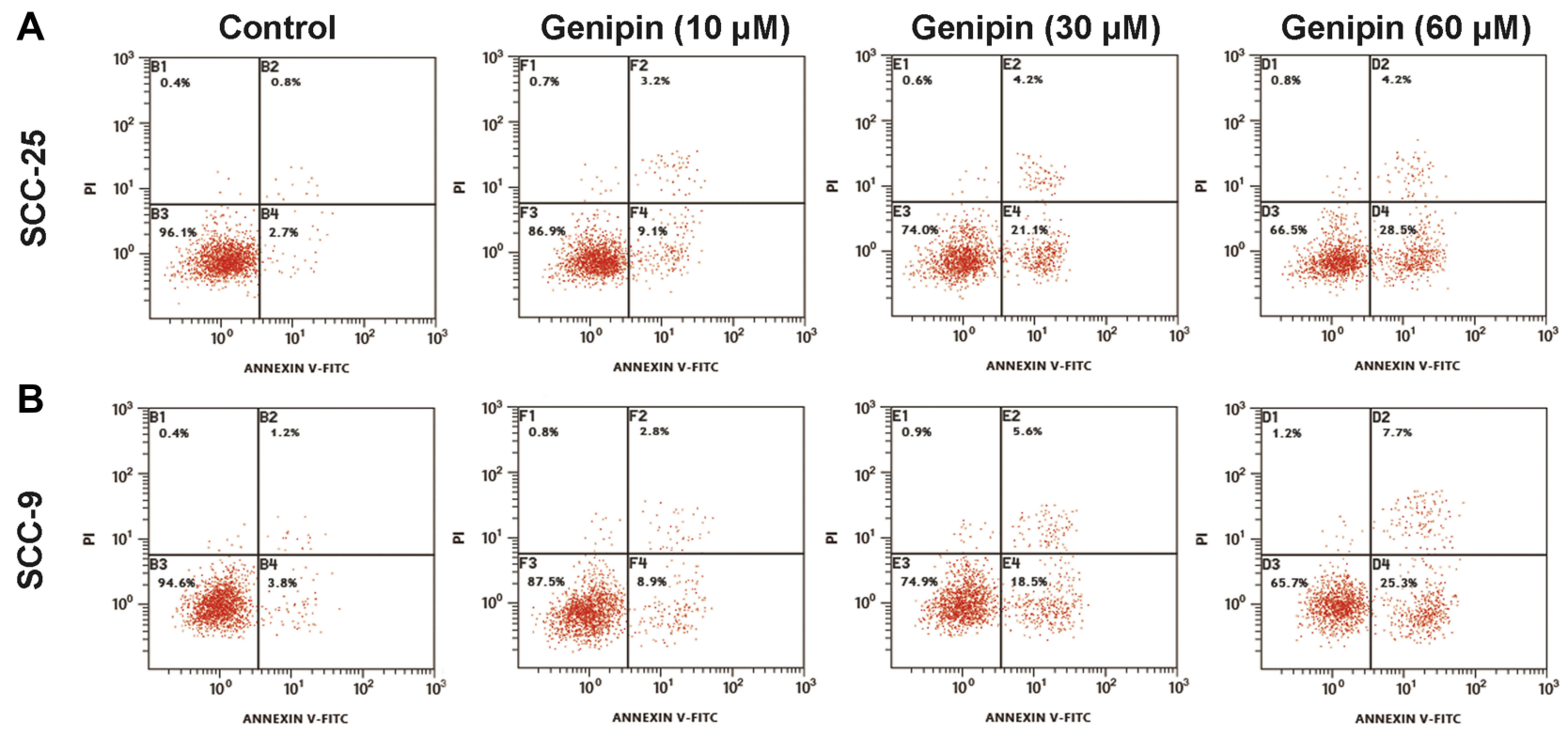

C

SCC-25
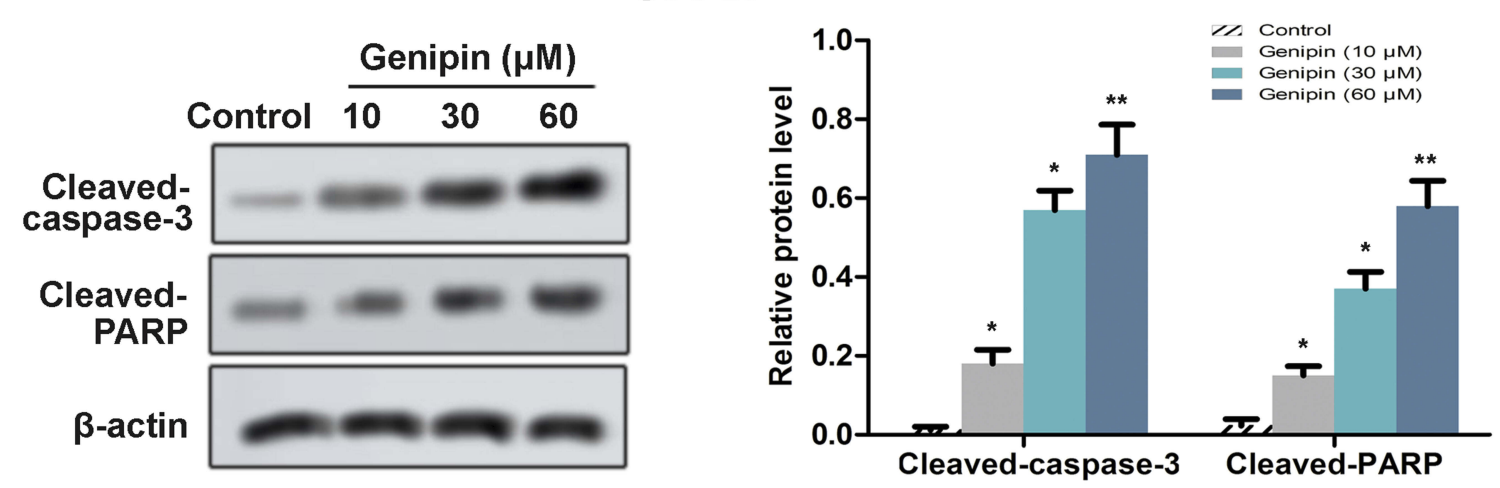

D

SCC-9
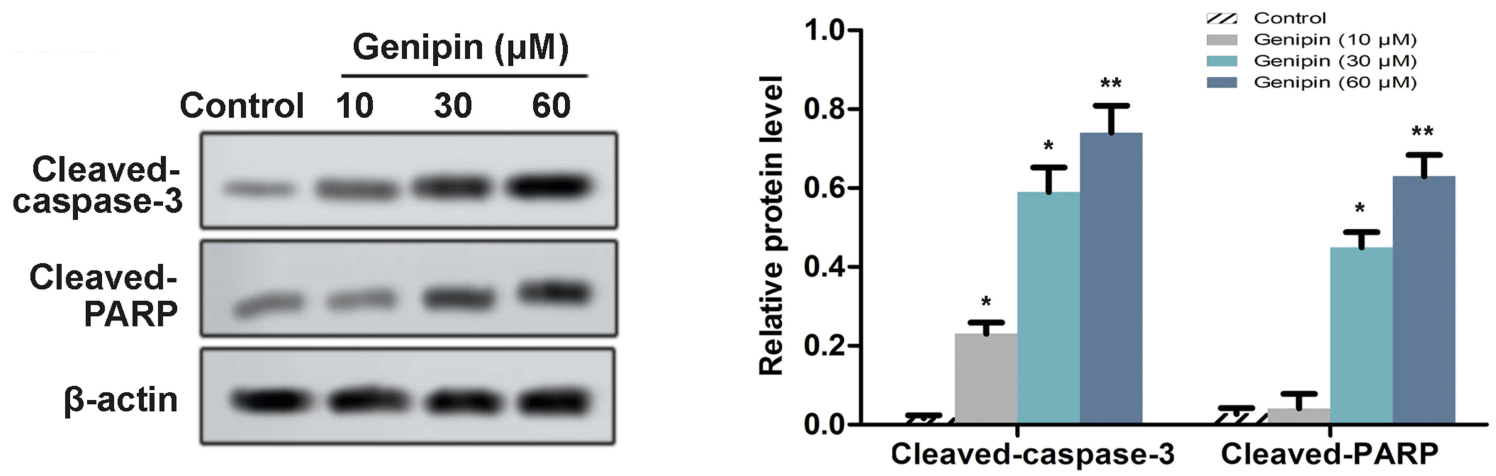

Figure 3 Effect of Genipin on cell apoptosis in OSCC. SCC-25 and SCC-9 cells were treated with three concentrations of Genipin (I0, 30 , and $60 \mu$ M). (A and B) SCC-25 and SCC-9 cells apoptosis were measured by flow cytometry. (C) The protein levels of cleaved-caspase-3 and Cleaved-PARP in SCC-25 were detected Western blotting. (D) The protein levels of cleaved- caspase- 3 and Cleaved-PARP in SCC- 9 were detected Western blotting. ${ }^{*} p<0.05$, ${ }^{* *} p<0.01$ compared with the control group. Data are presented as mean $\pm S D$, and all the experimental in triplicate.

protective function of Genipin for OSCC, our findings showed that Genipin inhibited the growth of OSCC cells, induced apoptosis, and improved the autophagy level, which provided a new strategy for the oral cancer treatment.
Genipin is derived from geniposide and is the major component of Gardenia jasminoides Ellis fruit. Li et $\mathrm{al}^{26}$ implicated that Genipin inhibited the cell growth in human bladder cancer. Jiang et $\mathrm{al}^{27}$ claimed that Genipin induced 
A
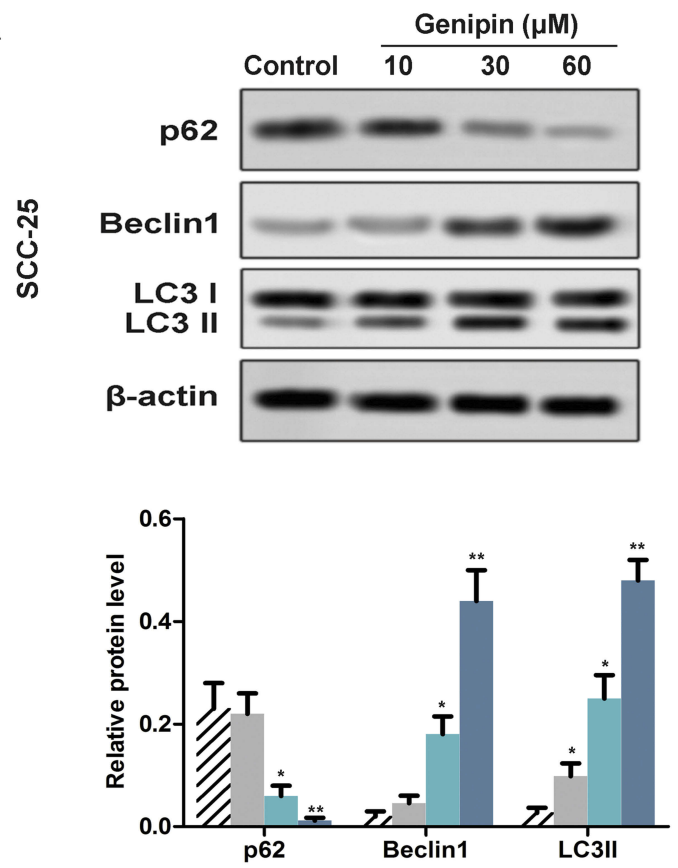

C

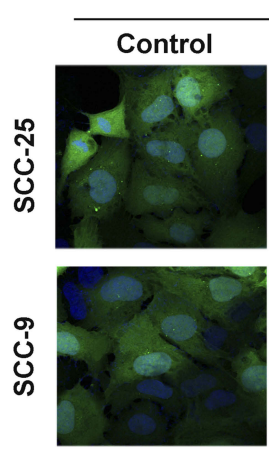

D
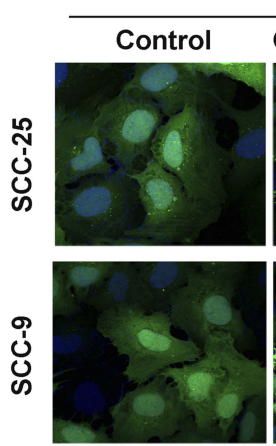

\section{LC3 Fluorescence}

Genipin $(10 \mu M)$ Genipin $(30 \mu M)$ Genipin $(60 \mu M)$
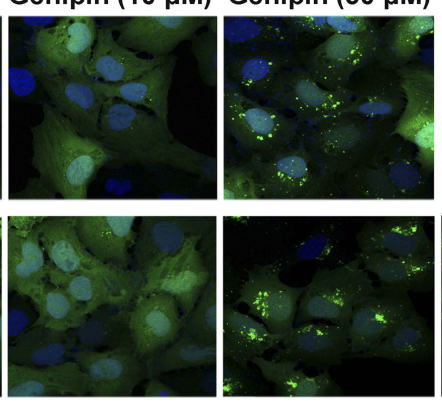

LC3 Fluorescence
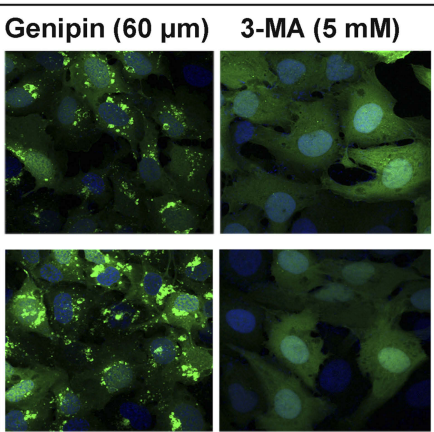

B

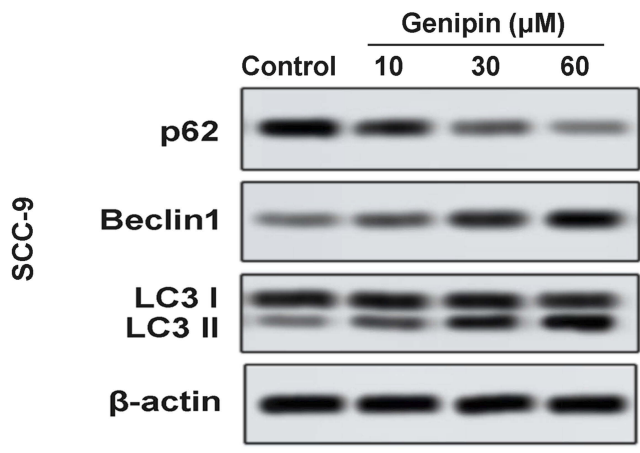

1/. Control

Genipin (10 $\mu \mathrm{M})$

- Genipin (30 $\mu \mathrm{M})$

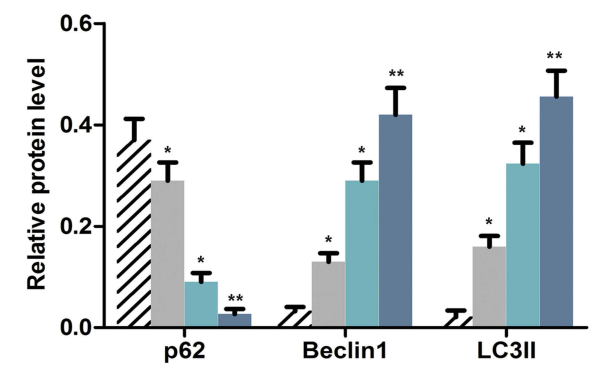

SCC-25

SCC-9

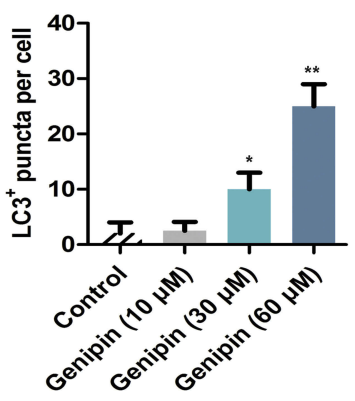

SCC-25

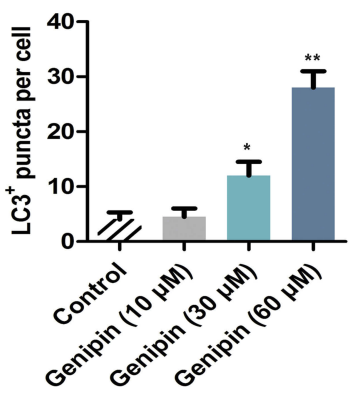

SCC-9

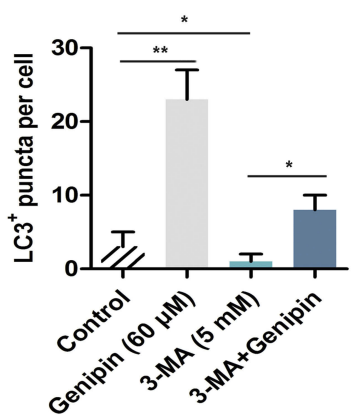

Figure 4 Effect of Genipin on cell autophagy in OSCC. SCC-25 and SCC-9 cells were treated with three concentrations of Genipin (I0, 30 , and $60 \mu \mathrm{M})$. (A) The autophagy relevant protein (P62, Beclin I, LC3II/LC3I) levels in SCC-25 were detected by Western blotting. (B) The autophagy relevant protein (P62, Beclin I, LC3II/LC3I) levels in SCC-9 were detected by Western blotting. (C) LC3 (LC3II) ${ }^{+}$puncta in SCC-25 and SCC-9 cells were measured by immunofluorescence assay, respectively. (D) After added the autophagy inhibitor 3-MA, LC3 (LC3II) ${ }^{+}$puncta in SCC-25 and SCC- 9 cells were measured by immunofluorescence assay, respectively. ${ }^{*}<<0.05$, $* *{ }^{2}<0.0$ I. Data are presented as mean $\pm \mathrm{SD}$, and all the experimental in triplicate.

HO-1 expression/activity and subsequently decreased vascular smooth muscle cell (VSMC) proliferation and migration. In our study, we found that Genipin inhibited the clone formation of SCC-25 and SCC-9 cells, and the expression of Ki67 and Survivin were down-regulation. Our animal experiments further confirmed the inhibitory effect of 
A

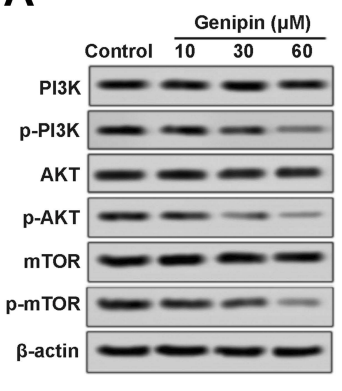

SCC-25
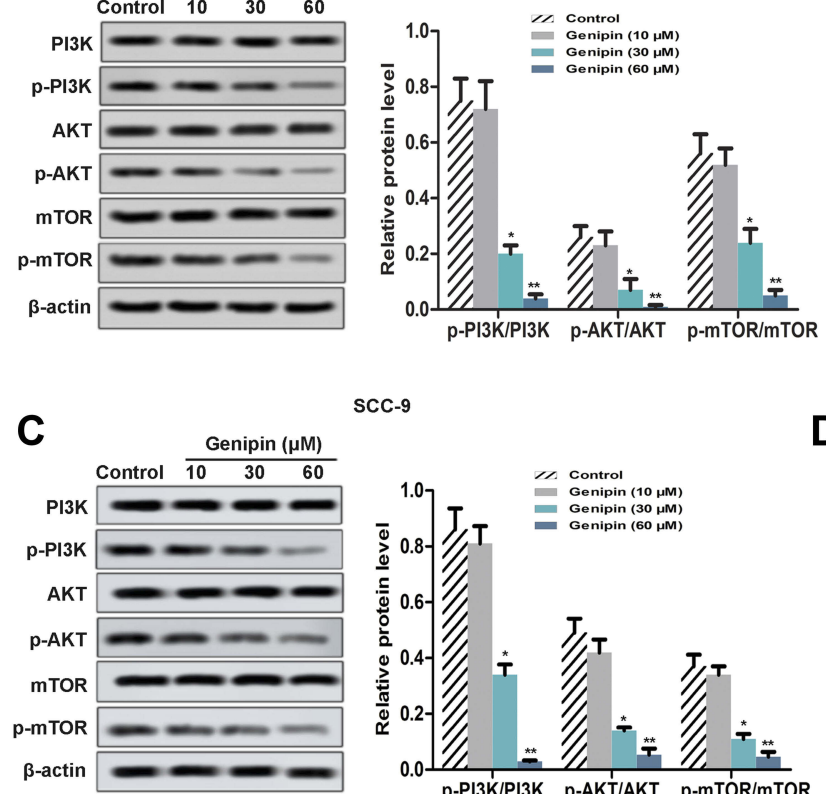

SCC-9

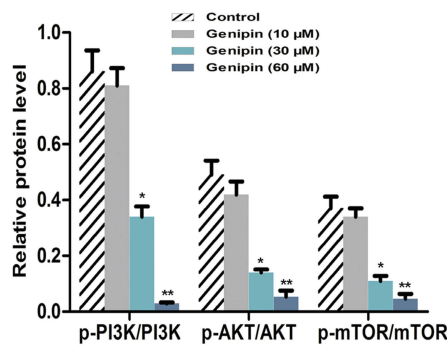

B

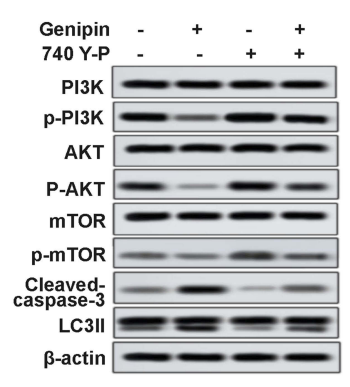

D

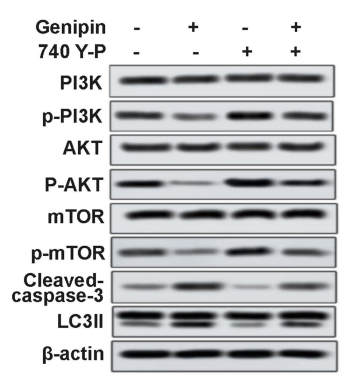

SCC-25

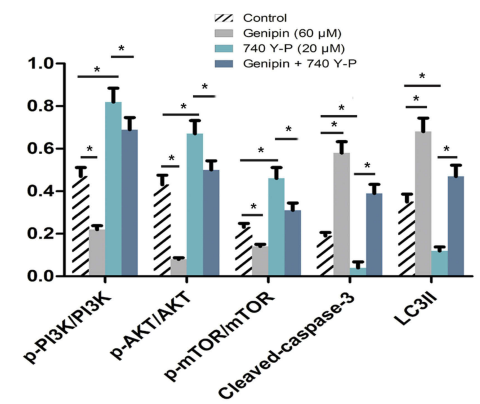

SCC-9

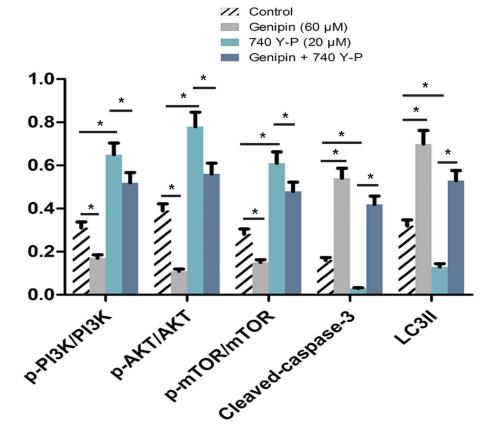

Figure 5 Regulating effect of Genipin on PI3K/AKT/mTOR pathway in OSCC. SCC-25 and SCC-9 cells were treated with three concentrations of Genipin (I0, 30, and $60 \mu \mathrm{M})$. (A) The expression of PI3K, p-PI3K, AKT, p-AKT, mTOR, and p-mTOR in SCC-25 were measured by Western blotting. (B) After added the PI3K activator 740Y-P, the expression of PI3K, p-PI3K, AKT, p-AKT, mTOR, p-mTOR, cleaved-caspase-3, and LC3II in SCC-25 were measured by Western blotting. (C) The expression of PI3K, p-PI3K, AKT, p-AKT, mTOR, and p-mTOR in SCC-9 were measured by Western blotting. (D) After added the PI3K activator 740Y-P, the expression of PI3K, p-PI3K, AKT, p-AKT, mTOR, p-mTOR, cleavedcaspase-3, and LC3II in SCC-25 were measured by Western blotting. ${ }^{*}<<0.05$, * ${ }^{*}<0.01$. Data are presented as mean $\pm S D$, and all the experimental in triplicate.

Genipin on tumor growth, the data showed that Genipin $(30 \mathrm{mg} / \mathrm{kg})$ treatment straightly reduced the size and weight of xenograft tumors, accompanied by a decrease in the expression of Ki67. Besides, the previous study demonstrated that Genipin treatment in human gastric cancer cell line induced apoptosis in a dose-dependent manner via p53independent Egr $1 / \mathrm{p} 21$ signaling pathway. ${ }^{28}$ In addition, the evidence also suggested that Genipin existed the anti-tumor activity of inducing apoptosis and inhibiting invasion in breast cancer. ${ }^{29}$ Exhilaratingly, in this study, the results showed that Genipin promoted the apoptosis of SCC-25 and SCC-9 cells in vitro and significantly raised the protein level of cleaved-caspase-3 and Cleaved-PARP. Similarly, we also found that Genipin promoted the expression of cleaved-caspase-3 in xenograft mouse model. Therefore, in combination with previous studies, we notarized that Genipin induced apoptosis in OSCC.

In cell biology, autophagy is a catabolic process of its own components by a lysosomal machine. ${ }^{30}$ Autophagy acts a key role in cell survival, which is a key pathway for homeostasis, development, and other pathophysiological processes. ${ }^{31}$ Moreover, autophagy showed an increase in the expression of autophagy-related proteins such as LC3II, Beclin-1, and ATG5, with a decrease in the expression of p62. ${ }^{32}$ Kinarivala et $\mathrm{al}^{33}$ reported that the activated Beclin-1 could induce autophagy. In this study, we investigated the effect of Genipin on autophagy in OSCC for the first time. In vitro, we found that Genipin treatment upregulated the protein levels of Beclin1 and LC3II, while down-regulated the protein level of P62. After coincubation with autophagy inhibitor 3-MA, the autophagy process was alleviated, compared with Genipin alone. Ulteriorly, animal experiments confirmed that Genipin induced an increase in the expression of LC3II protein. So, combined with the previous study, we suggested that Genipin induced autophagy in OSCC.

$\mathrm{PI} 3 \mathrm{~K} / \mathrm{AKT} / \mathrm{mTOR}$ signaling pathway has been widely known to be involved in the progression and tumorigenesis of many types of cancers ${ }^{34}$ including Oral Squamous cancer. ${ }^{35}$ There is sufficient evidence that $\mathrm{PI} 3 \mathrm{~K} / \mathrm{Akt} / \mathrm{mTOR}$ axis played an important role in the occurrence of oral cancer. ${ }^{36}$ For instance, Rizzo et $\mathrm{al}^{37}$ found that PI3K mutation was associated with the occurrence of oral squamous cell carcinoma. Prodromidis et $\mathrm{al}^{38}$ found that the up-regulation of Akt and 
A

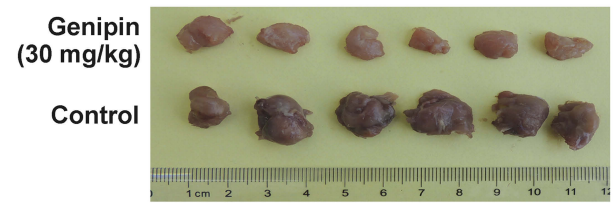

D

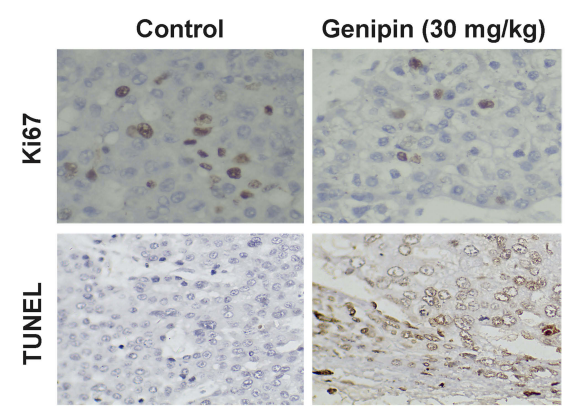

E

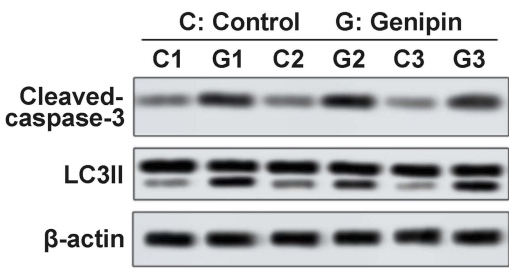

B
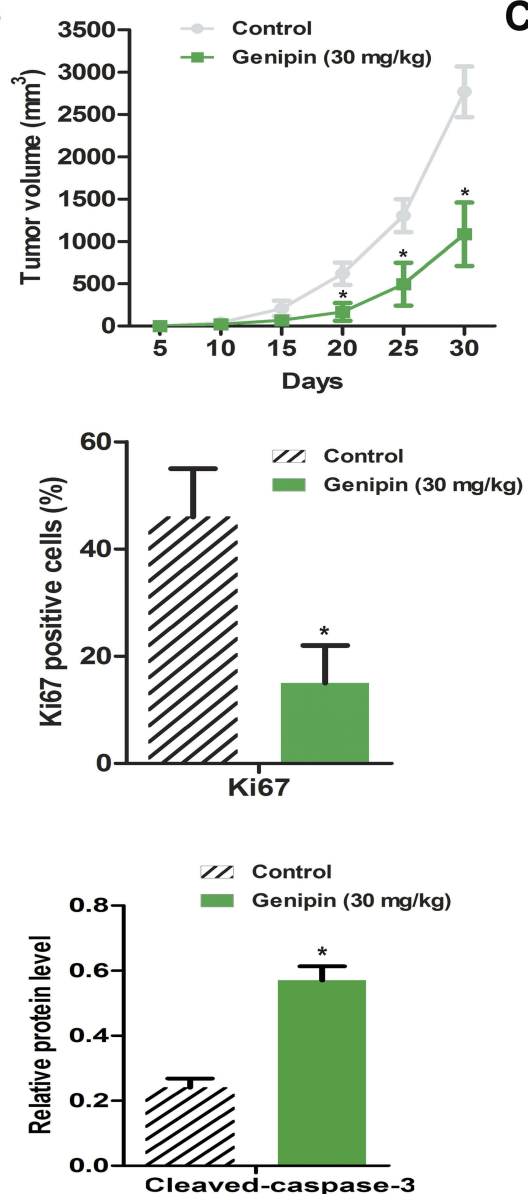

C
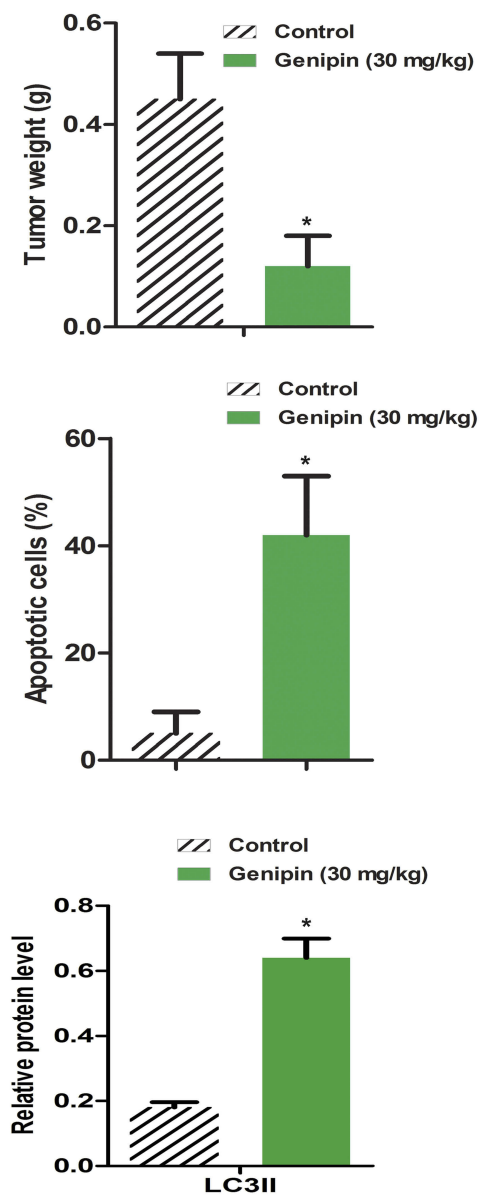

Figure 6 Genipin alleviated OSCC tumor growth in vivo. OSCC xenograft model was established with $30 \mathrm{mg} / \mathrm{kg}$ Genipin. (A-C) Tumor volume and tumor weights were measured. (D) The positive expression of Ki67 was detected by immunohistochemistry, the apoptosis of tumor tissues was measured by TUNEL assay. (E) The expression of cleaved- caspase-3 and LC3II in vivo were measured by Western blotting. ${ }^{*} \mathrm{p}<0.05$ compared with the control group. Data are presented as mean \pm SD, and all the experimental in triplicate.

mTOR expression in OSCC was more common than in oral lichen planus (OLP), which was thought to be the result of PI3K activation. Here, we found that Genipin inhibited the phosphorylation of PI3K, AKT, and mTOR in a concentrationdependent manner, indicating that the PI3K/Akt/mTOR signaling pathway was inactivated. In order to further verify the regulatory effect of Genipin in PI3K/Akt/mTOR, we added PI3K activator 740Y-P. As we predicted, 740Y-P increased the expression of p-PI3K, p-AKT, and p-mTOR, as well as the protein levels of cleaved-caspase-3 and LC3II. However, after co-treatment with Genipin and 740Y-P, Genipin weakened the activation of the pathway by 740Y-P. Therefore, it is believed that Genipin inhibits OSCC cells growth and improves the autophagy through suppressing PI3K/Akt/mTOR signaling pathway, and PI3K/Akt/mTOR pathway is an attractive target for the treatment of OSCC. ${ }^{39}$

Conclusively, this study showed that Genipin inhibited the cells' viability, induced apoptosis, and promoted autophagy in
OSCC, and its mechanism might be related to the inhibition of $\mathrm{PI} 3 \mathrm{~K} / \mathrm{Akt} / \mathrm{mTOR}$ signaling pathway. It provided a new candidate for OSCC drug treatment. Although we found that OSCC inhibited tumor growth in animal models, its protective mechanism in vivo needed further study.

\section{Disclosure}

The authors report no conflicts of interest in this work.

\section{References}

1. Ishida K, Tomita H, Nakashima T, et al. Current mouse models of oral squamous cell carcinoma: genetic and chemically induced models. Oral Oncol. 2017;73:16. doi:10.1016/j.oraloncology.2017.07.028

2. BH K, CW S, JK C, et al. HSPD1 repressed E-cadherin expression to promote cell invasion and migration for poor prognosis in oral squamous cell carcinoma. Sci Rep. 2019;9(1):8932. doi:10.1038/s41598019-45489-1

3. Ariyoshi Y, Shimahara M, Omura K, et al. Epidemiological study of malignant tumors in the oral and maxillofacial region: survey of member institutions of the Japanese society of oral and maxillofacial surgeons, 2002. Int J Clin Oncol. 2008;13(3):220-228. doi:10.1007/s10147-007-0756-9 
4. Prasad G, Mccullough M. Chemokines and cytokines as salivary biomarkers for the early diagnosis of oral cancer. Int $J$ Dent. 2013;2013:813756. doi:10.1155/2013/813756

5. Fu TY, Hou YY, Chu ST, et al. Manganese superoxide dismutase and glutathione peroxidase as prognostic markers in patients with buccal mucosal squamous cell carcinomas. Head Neck. 2011;33 (11):1606-1615. doi:10.1002/hed.21653

6. Warnakulasuriya S. Living with oral cancer: epidemiology with particular reference to prevalence and life-style changes that influence survival. Oral Oncol. 2010;46(6):407-410. doi:10.1016/j.oraloncology.2010.02.015

7. Song W, Tang Y, Qiao J, et al. The short-term safety evaluation of corneal crosslinking agent- genipin. Ophthalmic Res. 2019;62 (3):141-149. doi:10.1159/000499571

8. Li Y, Pan H, Li X, et al. Role of intestinal microbiota-mediated genipin dialdehyde intermediate formation in geniposide-induced hepatotoxicity in rats. Toxicol Appl Pharmacol. 2019;15(377):114624. doi:10.1016/j. taap.2019.114624

9. Somers P, De SF, Cornelissen M, et al. Genipin blues: an alternative non-toxic crosslinker for heart valves? J Heart Valve Dis. 2008;17 (6):682-688.

10. Inao M, Mochida S, Matsui A, et al. Japanese herbal medicine Inchin-ko-to as a therapeutic drug for liver fibrosis. J Hepatol. 2004;41(4):584-591. doi:10.1016/j.jhep.2004.06.033

11. Lee SY, Kim HJ, Oh SC, Lee DH. Genipin inhibits the invasion and migration of colon cancer cells by the suppression of HIF-1alpha accumulation and VEGF expression. Food Chem Toxicol. 2018;116 (Pt B):70-76. doi:10.1016/j.fct.2018.04.005

12. Ye J, Li J, Wang X, Li L. Medicinal supplement genipin induces p53 and Bax-dependent apoptosis in colon cancer cells. Oncol Lett. 2018;16(3):2957-2964. doi:10.3892/ol.2018.9025

13. Kim BR, Jeong YA, Na YJ, et al. Genipin suppresses colorectal cancer cells by inhibiting the sonic hedgehog pathway. Oncotarget. 2017;8(60):101952-101964. doi:10.18632/oncotarget.21882

14. Hennessy BT, Smith DL, Ram PT, Lu Y, Mills GB. Exploiting the PI3K/AKT pathway for cancer drug discovery. Nat Rev Drug Discov. 2005;4(12):988-1004. doi:10.1038/nrd1902

15. Carnero A, Blanco-Aparicio C, Renner O, Link W, Leal JF. The PTEN/PI3K/AKT signalling pathway in cancer, therapeutic implications. Curr Cancer Drug Targets. 2008;8(3):187-198. doi:10. 2174/156800908784293659

16. Jimenez C, Hernandez C, Pimentel B, Carrera AC. The p85 regulatory subunit controls sequential activation of phosphoinositide 3-kinase by Tyr kinases and ras. J Biol Chem. 2002;277:41556-41562. doi:10.1074/jbc. M205893200

17. Duran I, Kortmansky J, Singh D, et al. A Phase II clinical and pharmacodynamic study of temsirolimus in advanced neuroendocrine carcinomas. Br J Cancer. 2006;95:1148-1154. doi:10.1038/sj.bjc.6603419

18. Murugan AK, Munirajan AK, Tsuchida N. Genetic deregulation of the PIK3CA oncogene in oral cancer. Cancer Lett. 2013;338 (2):193-203. doi:10.1016/j.canlet.2013.04.005

19. Kim BR, Jeong YA, Na YJ, et al. Genipin inhibits the growth of human bladder cancer cells via inactivation of PI3K/Akt signaling. Oncotarget. 2017;8(60):101952-101964. doi:10.18632/oncotarget.21882

20. Omura K. Current status of oral cancer treatment strategies: surgical treatments for oral squamous cell carcinoma. Int J Clin Oncol. 2014;19(3):423. doi:10.1007/s10147-014-0689-z

21. Philips R, Martin D, Eskander A, et al. Effect of adjuvant radiotherapy treatment center volume on overall survival. Oral Oncol. 2018;78:46-51. doi:10.1016/j.oraloncology.2018.01.004

22. Ha J, Sung IY, Son JH, Stone M, Ord R, Cho YC. Analysis of speech and tongue motion in normal and post-glossectomy speaker using cine MRI. J Appl Oral Sci. 2016;24(5):472-480. doi:10.1590/1678-775720150421
23. Sroussi HY, Epstein JB, Bensadoun RJ, et al. Common oral complications of head and neck cancer radiation therapy: mucositis, infections, saliva change, fibrosis, sensory dysfunctions, dental caries, periodontal disease, and osteoradionecrosis. Cancer Med. 2017;6(12). doi:10.1002/cam4.1221

24. Kakoei S, Haghdoost AA, Rad M, et al. Xerostomia after radiotherapy and its effect on quality of life in head and neck cancer patients. Arch Iran Med. 2012;15(4):214-218.

25. Nyst HJ, Tan IB, Stewart FA, Balm AJ. Is photodynamic therapy a good alternative to surgery and radiotherapy in the treatment of head and neck cancer? Photodiagnosis Photodyn Ther. 2009;6 (1):3-11. doi:10.1016/j.pdpdt.2009.03.002

26. Li Zheng, Zhang T-B, Jia D-H, et al. Genipin inhibits the growth of human bladder cancer cells via inactivation of PI3K/Akt signaling. Oncol Lett. 2018;15(2):2619-2624. doi:10.3892/ol.2017.7588

27. Jiang F, Jiang $\mathrm{R}$, Zhu $\mathrm{X}$, Zhang $\mathrm{X}$, Zhan Z. Genipin inhibits TNF-alpha-induced vascular smooth muscle cell proliferation and migration via induction of HO-1. PLoS One. 2013;8(8):e74826. doi:10.1371/journal.pone.0074826

28. Ko H, Kim JM, Kim SJ, Shim SH, Ha CH, Chang HI. Induction of apoptosis by genipin inhibits cell proliferation in AGS human gastric cancer cells via Egr1/p21 signaling pathway. Bioorg Med Chem Lett. 2015;25(19):4191-4196. doi:10.1016/j.bmcl.2015.08.005

29. Kim ES, Jeong CS, Moon A. Genipin, a constituent of gardenia jasminoides ellis, induces apoptosis and inhibits invasion in MDA-MB-231 breast cancer cells. Oncol Rep. 2012;27(2):567-572. doi:10.3892/or.2011.1508

30. Kroemer G, Levine B. Autophagic cell death: the story of a misnomer. Nat Rev Mol Cell Biol. 2008;9:1004-1010. doi:10.1038/nrm2529

31. Bialik S, Dasari SK, Kimchi A. Autophagy-dependent cell death-where, how and why a cell eats itself to death. J Cell Sci. 2018;131:jcs215152. doi:10.1242/jcs.215152

32. Kim KH, Lee MS. Autophagy-a key player in cellular and body meta bolism. Nat Rev Endocrinol. 2014;10:322-337. doi:10.1038/nrendo. 2014.35

33. Kinarivala N, Patel R, Boustany R-M, Al-Ahmad A, Trippier PC. Discovery of aromatic carbamates that confer neuroprotective activity by enhancing autophagy and inducing the anti-apoptotic protein B-cell lymphoma 2 (Bcl-2). J Med Chem. 2017;60(23):9739-9756. doi:10.1021/acs.jmedchem.7b01199

34. Ediriweera MK, Tennekoon KH, Samarakoon SR. Role of the PI3K/ AKT/mTOR signaling pathway in ovarian cancer: biological and therapeutic significance. Semin Cancer Biol. 2019;59:147-160. doi:10.1016/j.semcancer.2019.05.012

35. Wang H, Deng X, Zhang J, et al. Elevated expression of zinc finger protein 703 promotes cell proliferation and metastasis through PI3K/ AKT/GSK-3beta signalling in oral squamous cell carcinoma. Cell Physiol Biochem. 2017;44(3):920-934. doi:10.1159/000485360

36. Vander Broek R, Mohan S, Eytan DF, Chen Z, Van Waes C. The $\mathrm{PI} 3 \mathrm{~K} / \mathrm{Akt} / \mathrm{mTOR}$ axis in head and neck cancer: functions, aberrations, cross-talk, and therapies. Oral Dis. 2015;21(7):815-825. doi:10.1111/odi.2015.21.issue-7

37. Rizzo G, Black M, Mymryk JS, Barrett JW, Nichols AC. Defining the genomic landscape of head and neck cancers through next-generation sequencing. Oral Dis. 2015;21(1):e11-e24. doi:10.1111/odi.2014.21. issue-1

38. Prodromidis G, Nikitakis NG, Sklavounou A. Immunohistochemical analysis of the activation status of the Akt/mTOR/pS6 signaling pathway in oral lichen planus. Int J Dent. 2013;2013(4):743456. doi:10.1155/2013/743456

39. Sonis ST, Amaral Mendes R. Could the PI3K canonical pathway be a common link between chronic inflammatory conditions and oral carcinogenesis? J Oral Pathol Med. 2016;45(7):469-474. doi:10.1111/ jop. 12436 


\section{Publish your work in this journal}

Drug Design, Development and Therapy is an international, peerreviewed open-access journal that spans the spectrum of drug design and development through to clinical applications. Clinical outcomes, patient safety, and programs for the development and effective, safe, and sustained use of medicines are a feature of the journal, which has also been accepted for indexing on PubMed Central. The manuscript management system is completely online and includes a very quick and fair peer-review system, which is all easy to use. Visit http://www. dovepress.com/testimonials.php to read real quotes from published authors.

Submit your manuscript here: https://www.dovepress.com/drug-design-development-and-therapy-journal 